\title{
Intervenciones para la atención de conductas adictivas de población femenil en centros de reinserción social
}

\author{
Interventions for the care of addictive behaviors in female social reintegration centers
}

Antonia Y. Iglesias Hermenegildo ${ }^{a}$, José Jesús Serrano Ruíz ${ }^{b}$

\begin{abstract}
:
Introduction: The consumption of psychoactive substances is a problem that has effect in both physical and emotional health of individuals. These repercussions have also an impact in public health, with an increase in morbidity and mortality rates associated to this problem. Objective: to identify and describe the interventions of addictive behavior in incarcerated women. Method: a systematic revision of PubMed, Ebsco, Dialnet and Redalyc data bases was carried out, as well as in public and government organizations documents, using the following key words: women, imprisonment, addictive behavior. Results: of the 14 scientific articles that were revised that include evidence of interventions for treatment of addictive behavior of incarcerated women, 11 of them conclude that these interventions are efficient, and 8 articles provide evidence that the damage reduction model is a highly effective and widely used model in Mexico for the treatment of this group. Conclusions: Mexico shows a lack of training and specialization of professionals capable of interventions in drug addiction related problems, besides the fact that economic resources for prevention and treatment programs are few.
\end{abstract}

Keywords:

Addictive behavior, intervention, prison, women.

\section{Resumen:}

Introducción: El consumo de sustancias psicoactivas ha tenido repercusiones en la salud física y psicológica de los individuos que las consumen, estas repercusiones se han visto reflejadas de igual forma en la salud pública, con el incremento en las tasas de morbilidad y mortalidad asociados a este consumo. Objetivo: Identificar y describir las intervenciones para la atención de conductas adictivas en mujeres en reclusión. Método: Se realizó una revisión sistemática, en las bases de datos PubMed, Ebsco, Dialnet y Redalyc, así como documentos emitidos por instituciones y organizaciones públicas y gubernamentales. Utilizando las siguientes palabras clave: mujeres, reclusión, conductas adictivas. Resultados: De los 14 artículos científicos revisados que reportan evidencia de las intervenciones para el tratamiento de conductas adictivas en mujeres en situación de reclusión, 11 concluyen que estas intervenciones son eficaces y ocho aportan evidencia de que el modelo de reducción de daños es uno de los más usados y efectivos en México para el tratamiento de este grupo poblacional. Conclusiones: En México, permean la falta de capacitación y especialización de profesionales para la intervención en temas de adicción a las drogas, además de que se destinan pocos recursos económicos a programas de prevención y tratamiento de las adicciones.

\section{Palabras Clave:}

Conductas adictivas, centros de reinserción, intervenciones, mujeres.

\section{Introducción}

El consumo de drogas ha tenido repercusiones en la salud física y psicológica del individuo que las consume, sin embargo, estas repercusiones se han visto reflejadas de igual forma en la salud pública, ya que se ha incrementado el consumo sobre todo de sustancias licitas, es decir, alcohol y tabaco, respecto al uso de drogas ilícitas; es difícil contar con cifras certeras sobre los niveles de consumo en la

\footnotetext{
a Autor de Correspondencia. Universidad Autónoma del Estado de Hidalgo, Instituto de Ciencias de la Salud, Pachuca, Hidalgo. México. ORCID: 0000-0002-4922-3884. Email: antonia_iglesias@uaeh.edu.mx

${ }^{\mathrm{b}}$ Universidad Autónoma del Estado de Hidalgo, Instituto de Ciencias de la Salud, Pachuca, Hidalgo. México. ORCID: 0000-0002-05256852 Email: jesus_serrano@uaeh.edu.mx
} 
población general, debido a que se considera su portación y uso como un delito, por lo que la mayoría de las personas no reporta su consumo, no es sino cuando la salud del consumidor se ve comprometida, por ejemplo en una sobredosis, que buscan atención médica y en algunos casos psicológica. Por lo que el consumo de drogas, sean lícitas o ilícitas, contribuyen al incremento de la mortalidad y discapacidad de la población, además de que el consumo de drogas se asocia directamente con aproximadamente sesenta enfermedades y lesiones relacionadas con el uso de violencia y accidentes. ${ }^{1}$

Las mujeres representan en el total global del sistema penitenciario una proporción menor, comparadas con los varones en todos los países, lo que convierte a la criminalidad en un problema de género. Sin embargo, la proporción de mujeres recluidas ha aumentado en la última década y cerca de $40 \%$ de las víctimas de los crímenes violentos también son mujeres. ${ }^{2}$

\section{Panorama epidemiológico del consumo de sustancias}

\section{Mundial}

Alrededor de 25 millones de personas entre 15 a 64 años en el mundo reportaron en el año 2015 haber consumido alguna sustancia ilegal por lo menos una vez en su vida, de acuerdo con datos del Informe Mundial Sobre las Drogas 2017. La droga que más se consume en el mundo es la marihuana que es consumida por aproximadamente 183 millones de personas, seguida de las drogas estimulantes y las anfetaminas con alrededor de 35 millones de consumidores misma cifra para los opioides. ${ }^{3}$

De acuerdo a los estudios realizados dentro del sistema penitenciario de E.U.A. en población femenina se sabe que entre las mujeres internas el abuso en el consumo de alcohol es 5 a 8 veces más frecuente, en comparación con la población general, y el abuso de sustancias ilícitas hasta 10 veces mayor. Siendo así que, las mujeres en reclusión o detención muestran una prevalencia para alteraciones por consumo de drogas adictivas de entre 30 a 52 por ciento, y para el trastorno por ingesta de alcohol de entre 17 y 24 por ciento. Otros hallazgos mencionan que el consumo de alcohol está presente hasta en $55 \%$ de las mujeres en reclusión. ${ }^{4}$

\section{Nacional}

Dentro del sistema penitenciario de México el consumo de drogas en mujeres en reclusión es un problema grave, para el que no se han tomado las medidas necesarias, ya que no existen programas adecuados para su atención. Las mujeres adultas privadas de su libertad oscilan entre 5\% y $7 \%$ de la población total recluida en México, sin embargo, para ellas no se tienen previstos los mecanismos para su futura reinserción social. ${ }^{4}$
Durante 2006 la población penitenciaria femenina era de 40 000; para 2010 se incrementó a 74000 internas. En cuanto a la distribución de la población penitenciaria por género, se registró un total de 11641 mujeres, lo que representa 4.8\% del total. Sólo 4189 , es decir, $35 \%$ se encuentran en centros de reclusión específicos para mujeres y 7,452, que representa el $65 \%$ se hallan en centros de reclusión mixtos. ${ }^{5}$ En el país existen 446 centros penitenciarios de los cuales, únicamente 10 son exclusivos para mujeres y 220 cuentan con anexos para esta población, lo que dificulta las condiciones de las mujeres y evidencia la falta de equidad respecto al género. ${ }^{2}$

En la Ciudad de México las cifras registran un aumento de tasas delincuenciales ya que en 1993 se registraron 446 mujeres, en 2003 incrementó a 1289, y para 2016 existían cerca de 1700 mujeres presas. $^{2}$

La prevalencia de esta problemática de salud pública es grave, ya que de acuerdo a lo reportado por un estudio realizado en mujeres recluidas en centros penitenciarios ubicados en la capital del país se reporta una prevalencia de trastornos por consumo de drogas ilícitas en 33\% y para trastornos relacionados con el alcohol un 37\%, cifras por demás elevadas que las de la población general. ${ }^{4}$

Por otro lado, respecto a las sustancias que más se consumen entre las mujeres que están en prisión, un estudio realizado con una muestra de 213 internas que se declaran usuarias de alguna droga, indicó a la cocaína como la droga más consumida, seguida de la marihuana, tabaco, sedantes, inhalables y tranquilizantes; del total de la muestra el $92 \%$ calificó positivamente a tres o más indicadores de dependencia a sustancias. ${ }^{6}$

El impacto del uso y abuso de sustancias se ve reflejado en los índices de mortalidad, pues de acuerdo con el Subsistema de Defunciones de la Dirección General de Información en Salud, reporta que los índices de mortalidad asociada al consumo de cualquier droga, entre el año de 1998 y el 2017 fue de 69,752 decesos de los cuales 4330 fueron mujeres. ${ }^{1}$

\section{Método}

Se llevó a cabo una revisión sistemática, en las bases de datos PubMed, Ebsco, Dialnet y Redalyc, así como documentos emitidos por instituciones y organizaciones públicas y gubernamentales.

Se establecieron los siguientes objetivos para la revisión bibliográfica:

Objetivos primarios: identificar aquellas intervenciones 0 programas para el tratamiento de conductas adictivas que consideran a la población general. Objetivos secundarios: identificar aquellas intervenciones para el tratamiento de conductas adictivas realizadas con población femenil de las prisiones.

Además de que se consideraron los siguientes criterios.

Criterios de inclusión: se consideraron únicamente aquellas intervenciones para el tratamiento de conductas adictivas realizadas en México con población en reclusión del año 
2000 a la fecha. Criterios de exclusión: intervenciones en población que no se encuentre en reclusión, estudios de caso.

\section{Resultados}

En México se han propuesto algunas intervenciones para el tratamiento de personas que consumen sustancias psicoactivas dentro de las prisiones, la tabla 1 muestra algunos de los programas propuestos y que cumplen con el objetivo primario de la investigación.

Tabla 1. Intervenciones para tratamiento de conductas adictivas en población en reclusión.

\begin{tabular}{|c|c|c|}
\hline Autor(es) año & $\begin{array}{l}\text { Nombre de la } \\
\text { intervención }\end{array}$ & Objetivo \\
\hline $\begin{array}{l}\text { La Secretaria de Salud, } \\
\text { Comisión Nacional Contra } \\
\text { las Adicciones (CONADIC), } \\
\text { 2015? }\end{array}$ & $\begin{array}{l}\text { Programa de Acción } \\
\text { Especifico: Prevención y } \\
\text { Atención Integral de las } \\
\text { Adicciones } 2013-2018 .\end{array}$ & $\begin{array}{l}\text { Establecimiento de politicas publicas dirigidas a } \\
\text { mejorar la calidad y ampliar la cobertura de los } \\
\text { servicios para prevenir y atender integralmente } \\
\text { problemas de salud ocasionados por el uso, abuso y } \\
\text { dependencia de sustancias. } \\
\text { Con enfasis en grupos de mayor riesgo, } \\
\text { incorporando un enfoque intercultural, de género y } \\
\text { de pleno respeto a los derechos humanos. }\end{array}$ \\
\hline $\begin{array}{l}\text { La Comision Nacional de } \\
\text { Derechos } \\
\text { (CNDH), } 2018{ }^{\text {H }} \text { Humanos }\end{array}$ & $\begin{array}{l}\text { Modelo de atención y } \\
\text { tratamiento para las } \\
\text { personas } \\
\text { farmacodependencia en } \\
\text { prisión. }\end{array}$ & $\begin{array}{l}\text { Atender desde el enfoque de la salud pública el } \\
\text { consumo de sustancias en prisiones, evitando la } \\
\text { estigmatización y discriminacion, garantizando los } \\
\text { derechos humanos de losindividuos. }\end{array}$ \\
\hline Castellanos, $2015 .^{9}$ & $\begin{array}{l}\text { Comunidad Terapéutica } \\
\text { (CT) }\end{array}$ & $\begin{array}{l}\text { Método que busca la rehabilitación de personas con } \\
\text { adicción a sustancias psicoactivas a través de la } \\
\text { modificación en el estilo de vida y del cambio de } \\
\text { identidad personal. Se sustenta en la ayuda mutua y } \\
\text { en la interacción comunitaria. }\end{array}$ \\
\hline NIDA, 2003.10 & & $\begin{array}{l}\text { Las CT son ambientes residenciales libres de droga } \\
\text { que usan un modelo jerarquico con etapas de } \\
\text { tratamiento que reflejan niveles cada vez mayores } \\
\text { de responsabilidadpersonaly social. }\end{array}$ \\
\hline
\end{tabular}

Respecto a las intervenciones para el tratamiento por conductas adictivas que se han realizado específicamente con mujeres que se encuentran en reclusión, la tabla 2 muestra aquellas intervenciones.

Tabla 2. Intervenciones para el tratamiento de conductas adictivas en mujeres en situación de reclusión.

\begin{tabular}{|c|c|c|}
\hline Autores & $\begin{array}{l}\text { Nombre de la } \\
\text { intervención }\end{array}$ & Resultados \\
\hline Castellanos, $2015 .^{9}$ & $\begin{array}{l}\text { Detección de drogas } \\
\text { y tratamiento de } \\
\text { adicciones en dos } \\
\text { centros } \\
\text { penitenciarios de la } \\
\text { CdMx }\end{array}$ & $\begin{array}{l}\text { Se trató de un estudio cualitativo que } \\
\text { indica que, son pocas las } \\
\text { intervenciones dentro de los centros } \\
\text { penitenciarios para el tratamiento de } \\
\text { las adicciones, pese a que es una de } \\
\text { las principales problemáticas de esta } \\
\text { población. }\end{array}$ \\
\hline $\begin{array}{l}\text { Romero, } \\
\text { Rodriguez, } \\
\text { Durand, } \\
\text { Colmenares } \\
\text { Saldivar, 2007. }{ }^{2}\end{array}$ & $\begin{array}{l}\text { Mujeres en prisión y } \\
\text { abuso de sustancias } \\
\text { adictivas }\end{array}$ & $\begin{array}{l}\text { Estudio transversal, en dos } \\
\text { CERESOS de mujeres, ubicados en } \\
\text { la CdMx. El problema de las } \\
\text { adicciones en mujeres en México no } \\
\text { ha sido una prioridad para las } \\
\text { politicas públicas y mucho menos el } \\
\text { de las pertenecientes a poblaciones } \\
\text { marginales. En este sentido, la } \\
\text { prisión es la respuesta a un problema } \\
\text { de salud. }\end{array}$ \\
\hline
\end{tabular}

Por otra parte, en México la mayoría de las intervenciones que se han realizado a mujeres que consumen sustancias psicoactivas en prisión, es bajo el modelo de reducción de daños; este modelo se define como el conjunto de ideas y estrategias prácticas enfocadas hacia la reducción de consecuencias negativas asociadas con el consumo de drogas, además de que, es también un movimiento por la justicia social y el respeto por, los derechos de las personas que usan drogas. ${ }^{11}$

A principios de la década de los 2000 se dio una de las investigaciones más importantes de México en torno al consumo de sustancias adictivas en mujeres ubicadas en centros de reclusión y la relación que podría tener con otros problemas de salud mental. Fue un grupo de investigadores del Instituto Nacional de Psiquiatría Ramón de la Fuente Muñiz (INPRFM) cuya finalidad fue crear una intervención desde la perspectiva de la reducción del daño y de género. ${ }^{12}$ Como resultado de dichas investigaciones se realizaron una serie de intervenciones con el modelo de reducción de daños, cuyos resultados se muestran en la tabla tres.

Tabla 3. Intervenciones para la reducción de daños por consumo de sustancias en mujeres en situación de reclusión en México.

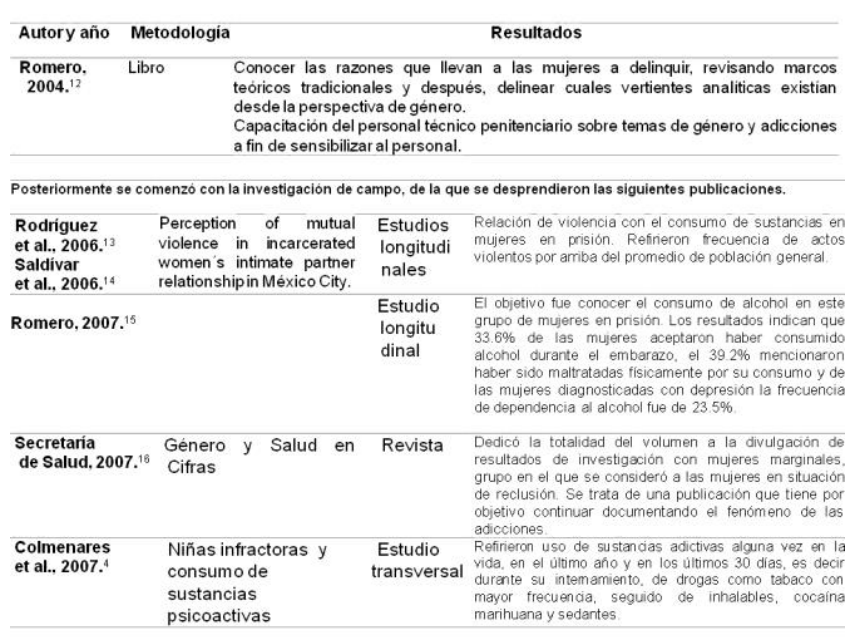

El problema de las adicciones en mujeres de México no ha sido una prioridad para las políticas públicas y mucho menos el de las pertenecientes a poblaciones marginales, como aquellas mujeres recluidas, en este sentido, la prisión es la respuesta a un problema de salud. ${ }^{2}$

Tras la revisión teórica y con base en los resultados preliminares del estudio es imperioso adoptar e implementar políticas públicas integrales orientadas a asegurar las condiciones de salud de las instituciones privatorias de la libertad. Dichas políticas deben estar orientadas a la prevención, diagnóstico y tratamiento oportuno de enfermedades, así como a la atención de grupos de mujeres en reclusión en particular situación de riesgo, por el uso, abuso y adicción a sustancias legales e ilegales.

Dentro de las distintas intervenciones, las que están basadas en la reducción del daño, por consumo de drogas, ponen especial énfasis a la reducción de consecuencias negativas del consumo de sustancias tanto para el sujeto que la consume y para su entorno, en vez de tratar de eliminar la problemática, ya que eso resulta bastante complejo dado que es un problema multifactorial. ${ }^{17}$

Las intervenciones para reducción del daño, son una de las propuestas para la atención de este grupo poblacional, cuyo objetivo consiste en aminorar las consecuencias negativas del consumo de sustancias psicoactivas. La intervención busca reducir riesgos relacionados con el consumo de drogas, y sus patrones de uso, así como mejorar la calidad de vida de los individuos. ${ }^{18}$ 
Este tipo de intervenciones presentan mejores resultados toda vez que el objetivo deja de ser únicamente la abstinencia en el uso de sustancias (que ya no se plantea como condición, sino como opción) pues se centra en disminuir los riesgos y los daños asociados al consumo.

Sin embargo, aun cuando se identifican resultados favorables y beneficio sobretodo en población femenil en condición de reclusión una de las principales dificultades para poner en marcha este tipo de estrategias es la falta de capacitación del personal dedicado a la atención de la población en centros de reclusión, de ahí la importancia de que se cuente con profesionales especialistas para la atención integral y multidisciplinaria de mujeres que presenten un problema de consumo de sustancias psicoactivas.

Los PRD más que como objetivo de un tratamiento pueden ser vistos como un acercamiento ético y pragmático a la problemática social de las drogas, que hace hincapié en reducir las consecuencias negativas del uso de sustancias más que en promover la abstinencia. ${ }^{19}$

La revisión teórica deja ver que puede contribuir para que aquellas mujeres que consumen drogas antes de su ingreso a alguna institución penitenciaria, y que pueden continuar con este consumo durante su internamiento, e incluso iniciarlo en la prisión. A través de estos programas se ofrece una opción de recibir la atención necesaria que coadyuve en el tratamiento de las adicciones y con que ello favorezca aminorar el daño físico, mental y social que esta problemática genera.

El aumento en el uso, abuso y adicción a sustancias adictivas legales e ilegales que hemos podido constatar a través de los datos epidemiológicos nos lleva a identificar este como un problema mundial que requiere de que tanto las instituciones como los profesionales mantengan una estrecha cooperación y coordinación respetando sus ámbitos de competencia.

Por otro lado, este tipo de intervenciones son muy complicadas no sólo por la falta de capacitación de quienes atienden a esta población a lo que se suman las características psicológicas, sociales y emocionales de la población femenil recluida en los centros de readaptación social.

Otro aspecto que es importante mencionar a pesar de lo delicado del mismo, es que se trastoca un ámbito delictivo que tiene que ver con el mercado de la venta de drogas en estos espacios. Ocurren riñas y agresiones físicas debido a que quienes consumen roban pertenencias para venderlas 0 empeñarlas y obtener dinero para pagar sus deudas o comprar más droga, además, una práctica común entre quienes distribuyen droga es que lesionen o maten a los internos que deben fuertes cantidades de dinero, como una medida de ejercer presión entre los deudores e intimidación para que paguen. Por otro lado, aunque no debería ocurrir, las autoridades y el personal de seguridad permiten la venta, posesión y el consumo de drogas a cambio de dinero. ${ }^{15}$ A pesar de este tipo de riesgos y complicaciones es necesario que en México y el mundo se realicen investigaciones que den cuenta de la problemática del consumo de drogas en los centros penitenciarios pues son pocas las investigaciones tanto a nivel nacional como internacional que ofrezcan datos confiables sobre el panorama epidemiológico del uso de sustancias en las prisiones, sobre todo cuando se trata de población femenil.
De igual manera son pocas las intervenciones con este tipo de poblaciones, llevarlas a cabo, permitirá conocer los factores asociados y generar estrategias en pro de la atención y el respeto de los derechos humanos de estos grupos poblacionales. Además de abrir espacios que posibiliten humanizar al sujeto, que se encuentra institucionalizado y en ocasiones cosificado por su condición de reclusión, las intervenciones son una oportunidad que les puede permitir a las mujeres reclusas una reflexión que las lleve a identificar y asumir su responsabilidad subjetiva en el consumo de sustancias.

Finalmente es necesario, reconocer que el problema mundial de las drogas sigue siendo una responsabilidad común y compartida que ha de abordarse desde una perspectiva multicausal y mediante una cooperación más intensa y efectiva que exige un enfoque integrado, multidisciplinario, equilibrado, amplio y basado en datos científicos.

\section{Conclusión}

En México, permean la falta de capacitación y especialización de profesionales para la intervención en temas de adicción a las drogas, además de que se destinan pocos recursos económicos a programas de prevención y tratamiento de las adicciones, hay un escaso desarrollo de programas gubernamentales bajo el modelo de reducción del daño y persiste el estigma y discriminación en torno al uso y abuso de sustancias, lo anterior, ofrece un campo de capacitación, actualización e intervención para la profesión desarrollando actividades de detección temprana, promoción y educación para la salud. ${ }^{11}$

Es así que, las intervenciones enfocadas en la reducción de daños sugieren un cambio de paradigma para la atención del uso problemático de drogas que puede convertirse en una adicción, ya que la meta del modelo es disminuir las consecuencias de la dependencia. ${ }^{20}$

\section{Referencias}

[1] Organización de los Estados Americanos El problema de las drogas en las Américas. [Internet]. Guatemala, Editor OEA; 2014. Disponible en: https://www.oas.org/docs/publications/LayoutPubgAGDrogas-ESP29-9.pdf [consultado 26 de marzo de 2020].

[2] INPRFM et al [Internet]. Encuesta Nacional de Consumo de Drogas, Alcohol y Tabaco, ENCODAT 2016-2017. . 2017 Disponible en: https://www.gob.mx/salud\%7Cconadic/acciones-yprogramas/encuesta-nacional-de-consumo-de-drogas-alcohol-ytabaco-encodat-2016-2017-136758 [consultado 18 marzo 2020].

[3] Romero, Rodríguez, Durand, Colmenares, Saldívar. Mujeres en prisión y abuso de sustancias adictivas. Género y salud en cifras. 2007; 5(1):130.

[4] Colmenares E, Romero M, Rodríguez E, Durand A, Saldívar, G. La doble marginalidad: niñas infractoras y consumo de sustancias psicoactivas. Género y salud en cifras.2007; 5(1):1-30.

[5] Gutiérrez JL. Accesibilidad del derecho a la salud de los grupos de población en los centros de reclusión. Revista dfensor. 2013; 8(6):0613.

[6] Romero M, et al. La reducción del daño por el abuso de drogas en las mujeres. En: Romero M, coord. Mujeres en prisión: una mirada a la salud mental. México: Liberadictus, A.C; 2004. 
[7] Secretaria de Salud. \& Comisión Nacional Contra las Adicciones (CONADIC). Programa de acción específico prevención y atención integral de las adicciones. México: Secretaría de salud; 2015.

[8] Comisión Nacional de los Derechos Humanos [CNDH]. Informe sobre los derechos humanos de las mujeres internas en centros de reclusión en México. Revista dfensor, 2013, 8(6), 46-52.

[9] Castellanos GE. Detección de drogas y tratamiento de adicciones en dos centros penitenciarios de la ciudad de México. ALFEPSI. 2015; 7(3): 93-104.

[10] National Institute of Drug Abuse. Preventing drug use among children and adolescents. A research-based guide for parents, educators, and community leaders. 2. ${ }^{\text {a }}$ ed. USA: Maryland: National Institutes of Health; 2003.

[11] Tizoc-Marquéz A, Rivera-Fierro K, Rieke-Campoy U, Cruz-Palomares M. Reducción de daños como estrategia para el uso y abuso de sustancias en México: Desafíos y oportunidades. Ra Ximbail. 2017; 13(2):39-51.

[12] Romero, M. et al. La reducción del daño por el abuso de drogas en las mujeres. En: Romero M, coord. Mujeres en prisión: una mirada a la salud mental. México: Servicio editorial Liberadicctus, A.C. 2004.

[13] Rodríguez E, Romero M, Durand A, Colmenares E, Saldívar G. Experiencias de violencia física ejercida por la pareja en las mujeres en reclusión. Salud Mental. 2006; 29(2)59-67.

[14] Saldívar G., Romero M., Rodríguez E, Durand A, Colmenares E. Perception of mutual violence in incarcerated women's intimate partner relationship in México City. International Journal of Prison Health. 2006; 2(1):35-47.

[15] Romero, Rodríguez, Durand, Colmenares \& Saldívar. Mujeres en prisión y abuso de sustancias adictivas. Género y salud en cifras. 2007; 5(1): $1-30$.

[16] Secretaría de Salud. [Internet]. Mujeres marginales. Género y cifras. 2007; 5(3): 1-50. Disponible en https://www.gob.mx/salud\%7Ccnegsr/documentos/revista-completagenero-y-salud-en-cifras-volumen-5-no-3-septiembre-diciembre-2007 [consultado 18 marzo 2020].

[17] Riley D. Harm reduction: concept and practice. A police discution paper. Substance use \& Misuse. 1999; 34(1): 09-24.

[18] IGIA. Contexto, sujetos y drogas. Un manual sobre drogodependencia. Disponible en http://www.grupigia.org/wpcontent/uploads/contextossujetosdrogas.pdf [consultado 04 de abril de 2020].

[19] Insúa P, Grijalvo J. Programas de reducción de riesgos en atención a las drogodependencias. Pap Psicól. 2000; 77(2). Disponible en: http://www.papelesdelpsicologo.es/vernumero.asp?id=844 [consultado 2 abril 2020].

[20] Velázquez BD, Friman RN, González GM. Programas de reducción de daños en las adicciones, un dilema ético. Correo Científico Médico. 2016; 20(4): 804-809. 